Revue internationale P.M.E.

Économie et gestion de la petite et moyenne entreprise

\title{
Apports et limites des théories contractualistes de la firme appliquées à la carrière des dirigeants de coopératives
}

\section{France Huntzinger et Anne Moysan-Louazel}

Volume 12, numéro 4, 1999

URI : https://id.erudit.org/iderudit/1008666ar

DOI : https://doi.org/10.7202/1008666ar

Aller au sommaire du numéro

Éditeur(s)

Presses de l’Université du Québec

ISSN

0776-5436 (imprimé)

1918-9699 (numérique)

Découvrir la revue

Citer cette note

Huntzinger, F. \& Moysan-Louazel, A. (1999). Apports et limites des théories contractualistes de la firme appliquées à la carrière des dirigeants de coopératives. Revue internationale P.M.E., 12(4), 77-100.

https://doi.org/10.7202/1008666ar
Résumé de l'article

Cet article propose une analyse du mode d'accès et du maintien au pouvoir des dirigeants de coopératives de production françaises, à la lumière du courant théorique contractualiste confronté aux résultats d'une enquête auprès d'une cinquantaine de p.-d. g. de sociétés coopératives de production (SCOP).

La théorie du marché interne du travail (MIT) est un cadre d'analyse pertinent pour étudier le déroulement de carrière interne ou transversale, qui privilégie la promotion interne dans le mode d'accès au pouvoir. La nécessité du recrutement externe du dirigeant suppose cependant l'ouverture du MIT. La théorie de l'enracinement fournit par ailleurs un éclairage intéressant sur les causes de l'existence des barrières à la sortie et le maintien au pouvoir des dirigeants qui développent des stratégies pour contourner les procédures de contrôle et augmenter leur latitude managériale.

Les pratiques managériales observées dans les SCOP françaises sont caractérisées par un fort taux de renouvellement des mandats des p.-d. g. et un recrutement externe lors de la mise en échec de la logique coopérative de promotion et de volontariat, ou sous la pression de la logique de la compétence. L'approche contractualiste conduit à négliger les effets de solidarités spécifiques à l'idéal coopératif tandis que les motifs d'enracinement sont diminués et justifiés dans les SCOP par l'efficacité de l'accumulation du capital humain, valeur ajoutée légitimant le pouvoir. La formation coopérative et l'éthique jouent le rôle de contre-pouvoirs permettant de limiter les effets pervers de l'enracinement, à l'origine du risque de banalisation du management coopératif.

Le dépassement du cadre d'analyse contractualiste privilégiant le phénomène d'opportunisme devient nécessaire pour tenir compte du rôle de la motivation du dirigeant de coopérative. Le recours au concept d'implication s'avère aussi pertinent pour expliquer celui de la culture et le phénomène d'« entrepreneur collectif » spécifique au statut de salarié-associé.
Ce document est protégé par la loi sur le droit d'auteur. L'utilisation des services d’Érudit (y compris la reproduction) est assujettie à sa politique d'utilisation que vous pouvez consulter en ligne.

https://apropos.erudit.org/fr/usagers/politique-dutilisation/ 


\title{
Apports et limites des théories contractualistes de la firme appliquées à la carrière des dirigeants de coopératives
}

France HUNTZINGER

Université du Maine

Anne MOYSAN-LOUAZEL

Université de Rennes I

\author{
MOTS CLÉS
}

Carrière - Marché interne - Enracinement - Dirigeant - Enquête Coopératives françaises - Implication - Solidarités

\begin{abstract}
RÉSUMÉ
Cet article propose une analyse du mode d'accès et du maintien au pouvoir des dirigeants de coopératives de production françaises, à la lumière du courant théorique contractualiste confronté aux résultats d'une enquête auprès d'une cinquantaine de p.-d. g. de sociétés coopératives de production (SCOP).

La théorie du marché interne du travail (MIT) est un cadre d'analyse pertinent pour étudier le déroulement de carrière interne ou transversale, qui privilégie la

\section{LES AUTEURS}

FRANCE HUNTZINGER est maître de conférences en gestion à l'Université du Maine et directrice de l'Institut universitaire professionnalisé (IUP) en management et gestion des entreprises de l'économie sociale Charles-Gide. Chercheure au GAINS, elle réalise depuis 15 ans des travaux sur le secteur des coopératives de production en France. Adresse: Université du Maine, Faculté de Droit, avenue Olivier-Messian, 72085 Le Mans, Cedex 9, France. Téléphone: 330243833534 ou 3126 . Télécopieur: 330243833562 . Courriel : france.huntzinger@ univ-lemans.fr

ANNE MOYSAN-Louazel est maître de conférences en économie à l'Université de Rennes I et chercheure au GAINS à l'Université du Maine. Ses travaux portent sur les théories des carrières et des motivations qu'elle a appliquées aux professions scientifiques (ingénieurs et chercheurs). Elle effectue actuellement des recherches sur le management des carrières des dirigeants de coopératives de production.
\end{abstract}


promotion interne dans le mode d'accès au pouvoir. La nécessité du recrutement externe du dirigeant suppose cependant l'ouverture du MIT. La théorie de l'enracinement fournit par ailleurs un éclairage intéressant sur les causes de l'existence des barrières à la sortie et le maintien au pouvoir des dirigeants qui développent des stratégies pour contourner les procédures de contrôle et augmenter leur latitude managériale.

Les pratiques managériales observées dans les SCOP françaises sont caractérisées par un fort taux de renouvellement des mandats des p.-d. g. et un recrutement externe lors de la mise en échec de la logique coopérative de promotion et de volontariat, ou sous la pression de la logique de la compétence. L'approche contractualiste conduit à négliger les effets de solidarités spécifiques à l'idéal coopératif tandis que les motifs d'enracinement sont diminués et justifiés dans les SCOP par l'efficacité de l'accumulation du capital humain, valeur ajoutée légitimant le pouvoir. La formation coopérative et l'éthique jouent le rôle de contre-pouvoirs permettant de limiter les effets pervers de l'enracinement, à l'origine du risque de banalisation du management coopératif.

Le dépassement du cadre d'analyse contractualiste privilégiant le phénomène d'opportunisme devient nécessaire pour tenir compte du rôle de la motivation du dirigeant de coopérative. Le recours au concept d'implication s'avère aussi pertinent pour expliquer celui de la culture et le phénomène d'« entrepreneur collectif » spécifique au statut de salarié-associé.

\begin{abstract}
This article analyses how directors of French production cooperatives attain and maintain power. It uses the insights of current contract theory to examine the results of a survey of about 50 directors of cooperatives.

The Internal Work Market theory, emphasizing internal promotion as a way into power, provides a relevant framework to study either internal or transverse career paths. However, the need to recruit directors externally suggests opening the Internal Work Market. Moreover, the theory of entrenchment sheds interesting light upon the causes of barriers to stepping down, and reveals how directors maintain power by developing strategies to get round the control procedures and to increase their managerial scope.

Managerial practices observed in French cooperatives are characterized by a high renewal rate of presidential mandates, and by recourse to external recruitment under the pressure of the need for competence, or whenever the cooperative logic of internal promotion and voluntary participation breaks down. The contractual approach tends to neglect the effects of solidarity specific to the cooperative ideal, whereas the grounds for entrenchment are both weakened and justified by the efficiency obtained through the accumulation of human capital, further vindicating the clinging on to power. A solid training in cooperatives and their ethics is a counterweight in limiting the perverse effects of entrenchment, and the risk of trivializing cooperative management.
\end{abstract}

In order to consider how motivation affects the director of a cooperative, it is necessary to go beyond the analytical framework of the contract theory, emphasizing 
the phenomenon of opportunism. Resorting to the concept of involvement also proves to be a relevant explanation of the culture and the phenomenon of "group entrepreneur " characterizing the status of the wage-earning senior partner.

\section{RESUMEN}

Este artículo propose un análisis sobre el modo de acceso y el mantenimiento del poder de los dirigentes de cooperativas de producción francesa a la luz de la corriente teórica contractualista confrontado con los resultados de una encuesta realizada a unos cincuenta presidentes directores generales de SCOP (Sociedades cooperativas de producción).

La teoría del Mercado Interno del Trabajo es un marco de análisis pertinente para estudiar el desarrollo de carrera interna o transversal que privilegia la promoción interna en el modo de acceso al poder. La necesidad de la contratación externa del dirigente supone sin embargo la apertura del MIT. La téoriá del arraigo ilustra por otra parte las causas de la existencia de barreras a la salida y el mantenimiento en el poder de los dirigentes que desarrolan estrategias para evitar los procesos de control y aumentar su latitud managerial.

Las prácticas manageriales observadas en las SCOP francesas se caracterizan por una fuerta tasa de renovación en los mandatos de los p.-d. g. y una contractación externa cuando fracasa la lógica cooperativa de promoción y de voluntariado a bajo la presión de la lógica de competencia. El punto de vista contractualista ignora los efectos de solidaridad especificos del ideal cooperativo mientras que los motivos de arraigo quedan disminuidos y justificados en las SCOP por la eficacidad de la acumulación del capital humano, valor anadido que legitima el poder. La formación cooperativa y la ética desempeñan el papel de contrapoderes que permiten limitar los efectos perversos del arraigo que está al origen del riesgo de trivialización del management cooperativo.

Hay que sobrepasar el marco de análisis contractualista que privilegia el fenómeno de oportunismo para tener en cuenta el papel de la motivación del dirigente de cooperativa. El recurso al concepto de implicación viene a ser tan pertinente para explicar el de la cultura y el fenómeno de empresario asociado especifico del estatuto de asalariado-asociodo.

\section{ZUSAMMENFASSUNG}

Auf der Basis einer Umfrage bei 50 Führungskräften wurde einerseits der Zugang zu Führungspositionen in französischen Genossenschaften analysiert und andererseits wurden Informationen erarbeitet, in welcher Form die Führungskräfte versuchen ihre Position zu halten.

Der interne Arbeitsmarktwert ist ein ständiger Analysefaktor, um den Ablauf einer Karriere zu studieren. Der interne Arbeitsmarktwert gibt Aufschluss über das Erreichen von internen Führungspositionen. Die Notwendigkeit einer externen Personalrekrutierung von Führungskräften setzt eine Bekanntgabe des internen Arbeitsmarktwertes voraus. Die sogenannte Verwurzelungstheorie gibt Aufschluss darüber, wie Führungskräfte Strategien und Kontrollinstrumente entwickeln, um ihr Managementpotential zu erkennen. 
Durch den zunehmenden Druck für Führungskräfte infolge struktureller, konkurrenzieller sowie strategischer Veränderungen im Sektor der Produktionsgenossenschaften konnte folgendes festgestellt werden: Die Führungskräfte in den Genossenschaften wechseln häufig ihre Arbeitsstelle und verstärken die Suche nach externen Kadermitarbeitern. Dieses Verhalten führt einerseits zu einer Vernachlässigung des genossenschaftlichen Gedankens, anderseits kann eine abnehmende Verwurzelung der Führungskräfte wegen des effizienten Erwerbs von personellen Humankapital konstatiert werden. Das genossenschaftliche Konstrukt sowie dessen Hintergrundgedanken spielen eine massgebende Rolle, um den Auswirkungen der kontraproduktiven Verwurzelung entgegenzuwirken.

Die Ueberschreitung der widersprüchlichen Rahmenbedingungen privilegieren ein opportunistisches Phänomen, welches sich in der Motivation der Führungskräfte der Genossenschaften widerspiegelt. Die Anwendung eines partizipativen Konzeptes zeichnet sich als sehr widerstandsfähig aus, um das Phänomen des "kollektiven entrepreneurs" und der Kultur eines partnerschaftlichen Entlöhnungssystems zu festigen.

\section{Introduction}

L'étude des carrières des dirigeants fait l'objet de nombreux travaux empiriques s'appuyant sur des outils méthodologiques variés. Alors qu'une forte majorité des travaux portent sur les carrières des dirigeants dans les grandes entreprises, leur carrière dans les petites et moyennes entreprises semble plus rarement étudiée.

Deux questions traversent notre travail : quel est le mode de production des dirigeants et quelles sont les stratégies de maintien au pouvoir?

Sous l'angle théorique, certaines approches de type contractualiste sont parties de ces interrogations. Notre objectif consiste dans cet article à montrer leur intérêt mais aussi leurs limites.

D'un point de vue empirique, notre recherche s'intéresse à un secteur particulier: le secteur des coopératives de production. Notre interrogation porte sur l'existence d'un marché interne du travail dans les entreprises coopératives de production, où les acteurs sont normalement réunis autour d'un idéal commun de « démocratie participative ». Notre problématique concerne la cohérence entre les règles constitutives du MIT et les spécificités de fonctionnement des SCOP ${ }^{1}$. Il s'agit donc pour nous de faciliter la compréhension des mécanismes à l'œuvre dans les SCOP pour assurer le renouvellement des dirigeants.

1. De nombreuses recherches existent sur le MIT, par exemple sur «MIT et stratégie du produit » développée par des chercheurs du Gains au Mans (Biencourt et al., 1997); mais, à notre connaissance, il n'y a pas de travaux sur la confrontation de ce concept à la firme coopérative. 
L'article tend à démontrer qu'il est nécessaire de dépasser le cadre d'analyse contractualiste où seule la coordination par les prix et les incitations est retenue, et de le compléter par des concepts étrangers à ce cadre d'analyse pour expliquer la réalité des pratiques coopératives qui, par bien des aspects, sont menacées de banalisation.

\section{Théories contractualistes de la firme et carrière des dirigeants}

Les deux facettes de notre approche théorique concernent respectivement les modes d'accès au poste de directeur général puis les mécanismes de son maintien à la tête de l'entreprise.

\section{Accès au pouvoir et théorie du marché interne du travail}

\subsection{La théorie du MIT}

La conception de l'entreprise comme MIT avait été annoncée dès les années 1950 et 1960 par des institutionnalistes américains tels que Kerr (1954) et Dunlop (1966). Au début des années 1970, les théoriciens du "dualisme du marché du travail » reprennent les travaux antérieurs et proposent de faire de l'entreprise l'acteur principal des relations de travail. Le marché interne du travail est un espace fermé et protégé au sens où les mécanismes d'allocation de la main-d'œuvre internes aux entreprises s'expliquent par des règles écrites ou non, et non pas par des variables économiques. C'est un espace de mobilité structuré en filières d'utilisation et de promotion des salariés. Ainsi, selon Doeringer et Piore (1971), les facteurs créateurs du MIT sont au nombre de trois : les compétences spécifiques, l'apprentissage sur le tas et la coutume, qui permettent aux salariés d'acquérir la mémoire collective de l'organisation, cette vaste encyclopédie de connaissances factuelles, de qualifications nées de l'habitude, de procédures de fonctionnement (Simon, 1980).

Néanmoins, le MIT et le marché externe sont interconnectés puisque le passage de l'un à l'autre s'effectue par des « ports d'entrée-sortie » correspondant à certains niveaux de classification de poste.

L'analyse microéconomique moderne s'est ensuite emparée de cette notion de MIT en lui attribuant trois fonctions essentielles : sélection, formation et incitation (Ballot et Piatecki, 1996). Elle analyse le MIT en termes d'individualisme méthodologique et d'efficience et perçoit l'entreprise uniquement comme un nœud de contrats entre les différentes parties. Cette approche est certainement satisfaisante pour analyser certains problèmes relatifs à la carrière des dirigeants. Cependant, 
une autre fonction du MIT, celle de l'intégration dans une communauté, n'est pas retenue et, à notre connaissance, jamais la création du MIT ne semble perçue comme l'aboutissement d'un projet, d'un idéal commun évinçant l'idée de pouvoir ou d'efficience.

\subsection{La notion de carrière}

Le marché interne du travail est un cadre d'analyse pertinent aussi bien pour étudier le déroulement de carrière interne à l'entreprise que pour repérer l'existence de carrière transversale à plusieurs entreprises, trajectoires rendues possibles par l'existence de ports d'entrée aux différents niveaux hiérarchiques. Ce concept nous paraît donc utile à l'étude des modes de production des dirigeants. Et, si les études de carrière portent souvent sur une comparaison entre une stratégie de mobilité entre entreprises et une stratégie de poursuite de carrière dans la même entreprise, nous nous intéressons ici à la fois à l'étude du modèle de carrière interne dans une organisation, c'est-à-dire aux parcours proposés aux salariés dans le système des positions internes, et au repérage des mobilités entre entreprises, puisque nous devons chercher à savoir si la carrière se poursuit dans plusieurs firmes.

Avec le changement dans les organisations qui se traduit par le passage d'un système hiérarchique à des réseaux décentralisés, c'est le fondement même du modèle traditionnel de carrière qui est remis en cause. La carrière, organisée comme une longue période de formation et de perfectionnement pour gravir les différents échelons hiérarchiques et accéder aux postes de dirigeants, n'est plus de mise dans des organisations donnant plus d'importance aux structures transversales et éliminant des niveaux hiérarchiques. Alors que l'on semble assister à la disparition des grosses sociétés intégrées verticalement au sein desquelles s'est construit le modèle hiérarchique de carrière, il est légitime d'analyser, d'une part, la transition vers un modèle de remplacement et, d'autre part, la prégnance éventuelle de ce modèle dans de plus petites structures. Récemment, Miles et Snow (1996) ont proposé une analyse des incidences des transformations des organisations sur les carrières. En faisant l'hypothèse que la carrière s'inscrit dans ou entre les organisations qui la structurent, nous souhaitons reprendre ce type d'analyse en liant notre réflexion sur la carrière des dirigeants à une réflexion plus large sur 1'organisation. C'est pourquoi, et suivant en cela Miles et Snow, nous considérons, d'une part, que « les formes organisationnelles conditionnent les compétences clefs requises pour pouvoir y participer», et, d'autre part, que «chaque forme organisationnelle comporte un mode spécifique de gestion des carrières, avec ses acteurs, ses logiques, ses mots d'ordre » (Cadin, 1997, p. 80-81). La carrière est une construction sociale qui s'appuie sur l'organisation, sa dimension collective et ses valeurs. 
Par ailleurs, nous utiliserons certains travaux menés sur la sociologie des dirigeants d'entreprise et leurs modalités d'accès aux postes de direction (Bauer et Bertin-Mourot, 1992). Le seul type de mobilité favorisé par le modèle organisationnnel de carrière, les mouvements verticaux de la main-d'œuvre basés sur l'existence d'une hiérarchie où l'ascension est la règle du jeu, est-il conforme à celui des dirigeants de coopérative ?

\subsection{Vers une typologie du dirigeant}

Caractériser les modes d'accès au poste de «numéro un » suppose de déterminer les critères d'arbitrage entre promotion interne et recrutement externe. Galambaud (1993) fait à cet égard la distinction entre cadres dirigeants et dirigeants. Les premiers gravissent les marches de la hiérarchie, les seconds arrivent dans l'entreprise pour immédiatement participer à sa direction et disposent d'une légitimité construite en dehors du mode de la firme d'accueil. De leur côté, Bauer et Bertin-Mourot (1992) offrent une typologie des grands patrons en cinq catégories, que nous pourrions adapter aux PME/PMI que sont les coopératives.

Les «montagnards» : partis du niveau jeune cadre ou en dessous, ils ont dû gravir, pour accéder aux fonctions suprêmes, de nombreux échelons en démontrant des capacités particulières. Les logiques de fonctionnement et de direction ont été progressivement acquises.

Les «héliportés » : ils sont partis d'un niveau déjà élevé de la hiérarchie. Leur poste d'entrée dans l'entreprise est proche du sommet et leur ascension est beaucoup plus courte.

Les «parachutés » : ils arrivent directement au sein de l'équipe dirigeante et ne connaissent pas la réalité de l'entreprise. On distingue le "parachuté catapulté », qui n'a jamais travaillé dans l'entreprise avant de prendre la direction du «parachuté non catapulté », qui entre comme membre de l'équipe dirigeante mais pas comme «numéro un».

Les « montagnards catapultés » : ils ont fait une vraie carrière dans une autre entreprise et, après avoir gravi tous les échelons, ont rejoint la firme en prenant directement la direction.

Si des barrières à l'entrée existent naturellement en fonction des critères de sélection qui valorisent non seulement les compétences mais aussi le capital humain spécifique et le réseau relationnel, il peut également apparaître des barrières à la sortie qui assurent aux dirigeants une impunité, une permanence, leur permettant de s'affranchir de toute référence à la réalité et à l'efficacité. Les théories récentes sur le gouvernement des entreprises fournissent, à notre avis, un éclairage intéressant sur les raisons de l'existence de ces barrières. 


\section{Maintien au pouvoir et théorie de l'enracinement}

\subsection{D'un comportement passif à un comportement actif d'un dirigeant}

Dans une entreprise organisée sous forme de société anonyme, la relation d'agence entre actionnaires et dirigeants est essentielle. Les intérêts des uns et des autres sont différents et peuvent entraîner des relations conflictuelles, voire un accaparement du pouvoir décisionnel par les dirigeants. L'étude de ces relations actionnairesdirigeants est ancienne puisque Berle et Means (1932) et plus tard Williamson (1964) étudiaient déjà le fonctionnement des firmes dites «managériales » où, selon eux, le pouvoir réel de décision appartient aux dirigeants qui possèdent à la fois l'information et la compétence.

La théorie de l'agence approfondit l'étude de cette relation en montrant « les limites d'une approche managériale faisant la part trop belle au pouvoir discrétionnaire [...]» (Glais, 1992, p. 47). L'entreprise est conçue comme un nœud de contrats où les relations, potentiellement conflictuelles, exigent la mise en place de moyens de contrôle de la délégation des droits de propriété. L'asymétrie d'information permet l'apparition de comportements opportunistes ${ }^{2}$ des agents et implique donc l'instauration des procédures de surveillance génératrices de coûts (d'agence).

Le contrat liant les actionnaires à des dirigeants constitue un contrat d'agence et selon Alchian et Demsetz (1972), puis Jensen et Meckling (1976) ou Fama (1980), le marché va être utilisé comme une pression internalisée, facteur de sanctions des dirigeants non respectueux des intérêts de l'actionnariat. D'une façon générale, l'accent est mis par ces auteurs sur l'identification des mécanismes de discipline des dirigeants supposés avoir désormais un comportement passif.

Or, pour Charreaux (1996), ces théories négligent trop le phénomène d'incomplétude des marchés et des contrats. Celui-ci accorde une importance déterminante à la notion de « latitude managériale », qui dépasse la seule dimension opportuniste de la théorie de l'agence, et qui définit l'existence d'un pouvoir discrétionnaire permettant au dirigeant de neutraliser les mécanismes disciplinaires issus du marché (financier et du travail). En effet, lorsque le dirigeant manipule l'information communiquée au marché, celui-ci ne peut plus être mobilisé comme moyen de pression sur les comportements. Cette mise en échec du marché, comme procédure de contrôle des dirigeants, redonne alors toute sa place à l'idée de l'entreprise gérée sous forme managériale.

2. Les individus sont opportunistes lorsqu'ils profitent de «la liberté qui leur est contractuellement accordée pour gérer les affaires de leur mandant dans une logique qui leur est plus favorable au détriment de ce dernier» (Gomez, 1996, p. 106). 
De nouvelles analyses posant l'hypothèse d'un comportement actif du dirigeant s'attachent alors à identifier les stratégies d'élargissement de leur latitude discrétionnaire, en particulier les stratégies « d'enracinement » dans leur entreprise. Pour Paquerot (1996), elles peuvent être définies comme des «stratégies mises en œuvre par les dirigeants pour modifier leur environnement (structures de contrôle, concurrence sur le marché de l'emploi, relations avec les partenaires, etc.) et accroitre leur pouvoir sur les actionnaires ainsi que sur les différents partenaires de la firme » (p. 213). Le dirigeant peut choisir de développer des actifs spécifiques qui mobilisent particulièrement son savoir-faire et sa capacité managériale de manière à rendre indispensable sa fonction dans l'entreprise. Gomez (1996) parle d'une «stratégie de spécification intentionnelle des actifs» (p. 113).

Les stratégies d'enracinement ont pour but d'augmenter les coûts de sortie et de contraindre les actionnaires à continuer le contrat malgré une certaine inefficience, en s'appuyant notamment sur deux facteurs de barrière à l'entrée de nouveaux dirigeants : l'asymétrie d'information et les contrats implicites avec les partenaires.

\subsection{Des modes d'enracinement variables au cours de la carrière de dirigeant}

En période active, on distingue trois phases d'enracinement différentes (Paquerot, 1996). La « valorisation » concerne le dirigeant nouvellement nommé, qui doit faire ses preuves. Celui-ci commencera par réaliser des investissements rentables puis pourra développer des contrats implicites avec les partenaires en jouant sur la complémentarité des actifs de la firme avec leur savoir-faire particulier. Son pouvoir est mérité et donc légitime puisqu'il permet d'améliorer la performance de la firme. Ensuite, le dirigeant tentera de réduire l'efficacité des contrôles qui s'exercent sur son activité en entreprenant des investissements destinés à accroître l'asymétrie d'information avec les partenaires (stakeholders), afin de disposer d'un espace de liberté plus grand. Enfin, la stratégie d'enracinement a pour objectif l'augmentation de la rémunération ou des avantages en nature, obtenus en général par la croissance interne ou externe de la firme, conférant un prestige certain. Les dirigeants ont toute latitude pour gérer l'entreprise à leur manière, pourvu qu'ils dégagent une rentabilité suffisante pour les partenaires. Ceux-ci ne seront pas incités à prendre des sanctions, car les coûts d'éviction deviennent importants, et il est même envisageable qu'ils n'aient pas conscience du comportement opportuniste du dirigeant tant l'asymétrie d'information est grande.

En fin de carrière, le dirigeant cherchera à conserver son pouvoir en obtenant de poursuivre son mandat au-delà de l'âge de la retraite. En raison des nombreux contrats implicites existant, la rupture serait trop coûteuse, notamment si les bénéficiaires (fournisseurs, clients, etc.) exigent d'officialiser ces contrats sous forme 
écrite pour que les avantages ou promesses se perpétuent après le remplacement du dirigeant actuel. Celui-ci s'opposera naturellement à la formalisation de ces contrats implicites afin de renforcer son pouvoir et de prolonger son mandat. Cette situation n'est pas nécessairement néfaste pour l'entreprise, car ce dirigeant peut rester encore très performant et très compétitif par rapport à des candidats à sa succession.

Quant à l'influence du dirigeant sur le choix du successeur, qui peut être assimilé à une continuation du contrat, l'asymétrie d'information va jouer un rôle central. Comme le remarque Paquerot (1996) «le transfert de l'information aux successeurs s'accompagne d'un transfert de pouvoir et donc d'un transfert d'enracinement des anciens dirigeants vers les nouveaux », qui bénéficient aussi d'un avantage concurrentiel sur les autres. En effet, cet auteur montre que «l'ancienneté relative des dirigeants, ainsi que la durée de leur mandat, influencent positivement la continuation de leur contrat après l'âge légal de la retraite » (p. 224), du fait de l'écart d'expérience qui peut exister entre le conseil d'administration et les dirigeants actuels.

\subsection{Les conséquences des stratégies d'enracinement}

Dans une première version, attribuée à Shleifer et Vishny (1989), les dirigeants cherchent à s'enrichir au détriment des actionnaires ou des partenaires et à réduire le risque d'être remplacés, au moyen de trois méthodes qui conduisent à l'enracinement. La première consiste à effectuer des investissements spécifiques, complémentaires de leur capital humain, qui leur permettent d'obtenir des rémunérations supérieures et rendraient coûteuse leur éviction par perte de valeur des actifs. La deuxième concerne la manipulation de l'information par les dirigeants qui ainsi peuvent choisir des investissements à visibilité réduite et accroître leur latitude discrétionnaire (Stiglitz et Edlin, 1992). La troisième se rapporte au contrôle des ressources : le dirigeant doit pouvoir se dégager de toute dépendance des apporteurs de ressources externes, notamment financières en privilégiant l'autofinancement, et représenter lui-même une ressource difficilement substituable.

Dans cette perspective qui suppose l'inefficacité, l'enracinement a des conséquences néfastes : politique d'investissement sous-optimale, choix d'activités trop ciblées, création d'un réseau relationnel personnel. Ainsi, ces stratégies d'enracinement, qui conduisent les dirigeants à augmenter leur pouvoir auprès de leurs partenaires indépendamment de la performance, aboutissent à des excès de pouvoir. «L'enracinement peut être perçu comme le fruit d'un pouvoir illégitime. » (Paquerot, 1996, p. 213) Cette approche, plutôt pessimiste, est-elle pertinente pour répondre à notre interrogation sur le comportement des dirigeants de SCOP ? Ceux-ci ont-ils tendance à s'enraciner selon les stratégies précédemment décrites? 
Dans une seconde version défendue par Castanias et Helfat (1992) et Garvey et Swan (1994), l'enracinement devient compatible avec l'efficacité, en se plaçant dans le cadre d'une approche de recherche des relations optimales entre dirigeant et firme.

De ce point de vue, il est plus important de protéger le capital managérial que de préserver la richesse des actionnaires, car les rentes liées aux compétences spécifiques à la firme pourraient faire l'objet d'une expropriation, en cas de remplacement des dirigeants actuels. Elles rémunèrent ainsi l'investissement en capital humain spécifique des dirigeants, qui seront incités à développer leurs capacités, à prendre des décisions créatrices de richesse, à entreprendre des investissements rentables. L'enracinement est considéré non plus comme une stratégie de neutralisation des systèmes de contrôle mais comme une procédure qui permet aussi de satisfaire les intérêts des actionnaires ou des partenaires. Cela implique l'existence et la préservation d'un espace discrétionnaire pour le dirigeant afin qu'il puisse créer les rentes attendues et réparties entre lui et les partenaires de la firme. Charreaux (1996) ajoute que, selon Garvey et Swan (1994), l'enracinement se justifie « comme un moyen d'internaliser les externalités qui caractérisent un monde où les contrats sont incomplets » (p. 56). Donc, le comportement d'enracinement et la recherche de latitude managériale n'ont pas de raison d'être jugés inefficaces, dans la mesure où les coûts engendrés par l'opportunisme des dirigeants sont plus faibles que ceux relatifs à l'absence de pouvoir discrétionnaire.

En conclusion, les références théoriques exposées dans cette première partie ont constitué la trame de notre démarche empirique dans le secteur des coopératives de production en France (SCOP), où leur validité sera testée dans ce contexte spécifique.

\section{Pratiques managériales des SCOP françaises}

La présentation de l'approche de terrain sera suivie des principaux résultats concernant la carrière de dirigeant dans les SCOP.

\section{Contexte de la recherche et méthodologie}

\subsection{Champ de la recherche}

Le champ de notre étude est très précis : que font les SCOP françaises en matière de production de leurs dirigeants et de leur maintien au pouvoir? Quelles sont les pratiques sur ce point? Sont-elles spécifiques et enracinées dans les valeurs coopératives?

L'existence de formes de participation propres à la coopérative (participation à la propriété, à la gestion et aux résultats de l'entreprise) nous a amenées à supposer 
que marché interne du travail et coopérative devaient intrinsèquement coexister. Y a-t-il cependant une dynamique particulière du marché interne du travail dans le milieu coopératif?

Selon les principes coopératifs de base, le sociétariat est essentiellement composé des travailleurs de la SCOP, la souscription obligatoire ou non de parts du capital social leur conférant, quel qu'en soit le montant, un droit de gestion sur une base démocratique (un homme égale une voix). Le pouvoir de décision est réparti entre les associés en vertu du principe de l'élection démocratique avec, comme corollaire, la circulation des mandats entre les membres.

La logique démocratique suppose que l'accès aux fonctions de direction dans une SCOP, conférant autorité et pouvoir, n'est pas une fin en soi, mais un moyen pour un coopérateur, à travers un parcours professionnel interne, de répondre à la logique d'usage qui caractérise ce type de firme, tant sur le plan individuel que collectif, dans l'optique d'une gestion permettant de satisfaire les besoins de ses membres. Dès lors, une contradiction n'apparaît-elle pas avec la logique du modèle traditionnel de carrière? Nous sommes au cœur du problème de la SCOP : la participation à la vie démocratique et l'accès aux postes de responsabilité et de direction sont fondés sur la liberté de choix et le volontariat, et pas seulement sur des mécanismes classiques de perspectives de carrière personnelle. La logique coopérative de promotion interne des membres pourrait ainsi se trouver contrecarrée par la logique démocratique du volontariat; des dysfonctionnements internes pourraient en résulter, en ce qui concerne notamment l'accès au pouvoir de direction de la SCOP.

Dans la coopérative, l'ascension au poste de dirigeant résulte-t-elle de compétences et de performances développées en son sein, qui justifieraient l'existence d'un parcours formateur pour acquérir ces compétences spécifiques et la mémoire collective ? La carrière interne avec entrée au bas de la hiérarchie suivie d'une progression donnant accès aux fonctions de dirigeant existe-t-elle ? S'appuie-t-elle sur la logique coopérative de solidarité et de partage ou sur une logique individualiste d'accès aux plus hautes fonctions plus traditionnelle?

Le problème que nous soulevons implicitement ici est finalement celui de la confrontation de deux logiques organisationnelles divergentes. La coopérative qui repose sur des principes participatifs et démocratiques peut-elle accueillir des carrières dont le modèle repose traditionnellement sur des principes hiérarchiques et bureaucratiques?

\subsection{Méthodologie}

L'objectif est de faire apparaître la façon dont se construit l'autorité légitime dans les coopératives en tentant de mesurer la part du modèle de l'autoproduction par rapport au modèle de la sous-traitance, d'une part, et d'étudier le comportement 
du dirigeant pour développer une véritable carrière en briguant le renouvellement de nouveaux mandats, d'autre part. Pour répondre à ces problématiques, nous avons opté pour une double approche.

Tout d'abord, nous avons choisi de réaliser une enquête postale auprès des dirigeants actuels des SCOP françaises. Dans la mesure où l'existence du MIT se révèle dans des organisations d'une certaine taille structurée en plusieurs niveaux hiérarchiques autorisant une perspective de déroulement de carrière, le questionnaire a été adressé aux SCOP employant 50 salariés et plus, soit 108 coopératives en 1996 ( $8 \%$ du nombre total). Un taux de réponse au questionnaire de $50 \%$ peut être considéré comme tout à fait satisfaisant.

Ensuite, ce travail sera complété par une enquête qualitative par interview en cours auprès de 10 cas de président-directeur général.

Seuls les résultats de l'enquête postale seront ici exposés.

\subsubsection{Typologie des questions}

La plupart sont de type fermé ou à choix multiples pour permettre un traitement informatisé, et portent, pour une part, sur l'entreprise (historique, activité, statut, etc.), pour l'autre part, sur le dirigeant actuel lui-même (origine, formation, motivation, etc.), en vue d'une analyse croisée. Les questions ouvertes se rapportent essentiellement aux fonctions successives exercées et à la formation professionnelle.

\subsubsection{Caractéristiques de l'échantillon de SCOP étudiées}

Le profil démographique et économique de celui-ci est le reflet, partiel certes, des évolutions historiques et de la capacité d'adaptation à la crise de l'ensemble du Mouvement coopératif de production (Huntzinger, 1994).

TABleau 1

Les SCOP par secteur d'activité

\begin{tabular}{lccccc}
\hline & $\begin{array}{c}\text { Bâtiment et } \\
\text { et travaux } \\
\text { publics }\end{array}$ & $\begin{array}{c}\text { Activités } \\
\text { industrielles }\end{array}$ & $\begin{array}{c}\text { Services } \\
\text { matériels }\end{array}$ & Imprimerie & $\begin{array}{c}\text { Services } \\
\text { intellectuels } \\
\text { et culturels }\end{array}$ \\
\hline Ensemble SCOP & $36 \%$ & $19 \%$ & $15 \%$ & $8 \%$ & $22 \%$ \\
Échantillon & $42 \%$ & $30 \%$ & $15 \%$ & $8 \%$ & $6 \%$ \\
\hline
\end{tabular}

Moins de la moitié, contre $75 \%$ pour l'ensemble, sont nées après 1978 , début de la période de boom démographique (leur nombre ayant été multiplié par 2,5 en cinq ans), et $21 \%$ datent d'avant la Deuxième Guerre mondiale. 
TABLEAU 2

Les SCOP par taille

\begin{tabular}{lccc}
\hline & $\mathbf{5 0}$ à $\mathbf{8 0}$ salariés & $\mathbf{8 0}$ à $\mathbf{1 5 0}$ salariés & $\mathbf{1 5 0}$ salariés et plus \\
\hline Échantillon & $42 \%$ & $25 \%$ & $33 \%$ \\
\hline
\end{tabular}

Sur le plan juridique, compte tenu de l'obligation d'adhérer pour les nouveaux salariés après deux ans d'ancienneté ( $75 \%$ des cas), le taux de sociétariat dépasse les $80 \%$ dans $60 \%$ des SCOP étudiées, avec une évolution positive de 10 points depuis 10 ans, et cela, malgré le fait qu'en général les SCOP de bâtiment mobilisent moins de sociétariat que les autres secteurs d'activité. Le statut juridique adopté est celui de société anonyme (96\%) avec conseil d'administration et p.-d. g. La représentation au CA n'est généralement pas proportionnelle aux catégories socioprofessionnelles, au nom de la liberté et de l'égalité des individus ; néanmoins, une sur quatre a instauré ce système de quota, afin de stimuler la participation de toutes les catégories de sociétaires et sans doute d'éviter la monopolisation du pouvoir par un groupe. Enfin, la durée des mandats est presque toujours limitée à trois ou quatre ans, mais comme ils sont renouvelables, il n'y a pas de véritable garantie statutaire de circulation des mandats entre les membres.

\section{Principaux résultats}

\subsection{Portraits des dirigeants des SCOP étudiées}

Ce sont des hommes, dont l'âge moyen est de 50 ans; la moitié d'entre eux ont entre 45 et 55 ans et un quart entre 55 et 65 ans. La moitié a fait partie du groupe des fondateurs de leur SCOP, en corrélation avec le fait que la moitié de notre échantillon s'est constituée après 1978 et que la durée moyenne de travail dans celle-ci est de 15 ans.

En général, l'accession au poste de p.-d. g. a été précédée d'une période de participation au conseil d'administration (62\% des cas). Cependant, $36 \%$ de ces p.-d. g. ont exercé cette fonction sans étape préalable au sein de l'instance élective, dont $28 \%$ sont devenus p.-d. g. dès leur entrée dans la coopérative, élus lors de la création parmi les membres du groupe de fondateurs. Dans ces cas-là, l'occasion de poursuivre une carrière de type classique est impossible, mais le maintien du dirigeant au poste de p.-d. g. grâce au renouvellement des mandats par les membres coopérateurs du conseil d'administration permet d'évoquer l'existence d'une « carrière de dirigeant». En effet, ces dirigeants sont à leur poste de p.-d. g. depuis neuf ans en moyenne; certains y sont même depuis plus de 14 ans (20\%); quoique $38 \%$ assument cette fonction depuis moins de quatre ans, s'agissant de leur premier mandat, on constate pour les autres un taux de renouvellement important. 
TABLEAU 3

Les étapes dans l'ascension au poste de dirigeant

\begin{tabular}{lcccc}
\hline Accès direct & $\begin{array}{c}\text { Un poste } \\
\text { de direction } \\
\text { fonctionnelle }\end{array}$ & $\begin{array}{c}\text { Deux postes à } \\
\text { responsabilité } \\
\text { croissante }\end{array}$ & $\begin{array}{c}\text { Trois postes à } \\
\text { responsabilité } \\
\text { croissante, } \\
\text { et plus }\end{array}$ \\
\hline $\begin{array}{l}\text { Dirigeants } \\
\text { de l'échantillon }\end{array}$ & $30 \%$ & $30 \%$ & $25 \%$ & $15 \%$ \\
\hline
\end{tabular}

TABLEAU 4

Mode d'accès au pouvoir des dirigeants de SCOP

\begin{tabular}{lcccc}
\hline & «Montagnards » & «Héliportés » & «Parachutés » & $\begin{array}{c}\text { «Montagnards } \\
\text { catapultés » }\end{array}$ \\
\hline $\begin{array}{l}\text { Dirigeants } \\
\text { de l'échantillon }\end{array}$ & $10 \%$ & $50 \%$ & $19 \%$ & $21 \%$ \\
\hline
\end{tabular}

En dehors des situations où le dirigeant actuel est devenu p.-d. g. directement dès son arrivée, la personne a occupé une ou plusieurs fonctions préalables, situées le long d'un parcours hiérarchique et d'apprentissage sur le tas. Cela permet-il de confirmer l'existence d'un réel déroulement de carrière au sein de la SCOP dans le cadre d'un MIT?

\subsection{Les facteurs du déroulement de carrière}

Il dépend non seulement de l'ancienneté de la personne dans l'entreprise mais aussi de l'âge de la coopérative. Par conséquent, seules les SCOP les plus anciennes (dont la création remonte à au moins 15 ans) font apparaître des possibilités d'accès au pouvoir après plusieurs étapes correspondant à des responsabilités de plus en plus étendues. Mais seulement $9 \%$ des p.-d. g. de l'échantillon ont mené toute leur carrière professionnelle dans leur SCOP actuelle. Nous pouvons alors élargir le repérage du MIT au milieu coopératif, dans la mesure où $13 \%$ des dirigeants actuels ont auparavant travaillé dans d'autres SCOP ou d'autres types de coopératives (par exemple, agricole).

TABLEAU 5

Ancienneté des dirigeants dans la SCOP

\begin{tabular}{lccc}
\hline & Moins de 10 ans & De 10 à 15 ans & Plus de 15 ans \\
\hline Échantillon & $30 \%$ & $30 \%$ & $40 \%$ \\
\hline
\end{tabular}


Ainsi, à l'exception des SCOP créées récemment, l'accès au pouvoir supposerait une présence d'au moins 10 ans dans la coopérative, ce qui est le cas de $70 \%$ des p.-d. g. actuels. Ce délai peut-il être jugé suffisant pour évoquer la notion de carrière interne ? Par ailleurs, nous remarquons que $89 \%$ des personnes interrogées ont travaillé dans une entreprise classique avant d'intégrer une SCOP, et $21 \%$ y exerçaient déjà de hautes fonctions de direction. Donc, parmi les dirigeants actuels, y compris ceux qui ont accédé directement au poste de p.-d. g., figurent des personnes ayant déjà fait carrière hors SCOP.

La détection d'un MIT passe aussi par l'étude du rôle de la formation dans le déroulement de carrière et l'accès au pouvoir. À cet égard, l'enquête montre que $66 \%$ des dirigeants se déclarent diplômés et que $53 \%$ se considèrent comme autodidactes, en ce sens que les diplômes acquis l'ont été au cours de leur vie professionnelle. La plupart d'entre eux ont suivi des formations au cours de leur carrière dans la SCOP : $68 \%$ une formation à la gestion, $45 \%$ une formation technique et $34 \%$ une formation à la vie coopérative. Néanmoins, plus de la moitié des personnes interrogées déclarent que cela n'a rien changé directement à leur situation personnelle ou professionnelle. Seulement $25 \%$ répondent positivement, en précisant que la formation à la gestion a joué un rôle majeur (50\% des cas), tandis que la formation coopérative n'est déterminante que pour un quart d'entre eux. Un peu plus de la moitié des dirigeants pensent tout de même que ces formations ont contribué indirectement à leur accès au pouvoir de direction.

\subsection{L'ouverture du MIT et l'idéal coopératif}

Le MIT est une structure hiérarchisée d'emplois pourvus par promotion, à l'exception de l'échelon le plus bas. L'observation des flux d'entrée dans les coopératives de notre échantillon montre que promotions et recrutements externes peuvent coexister pour l'accès au poste de dirigeant. Les coopératives embauchent à la fois aux échelons intermédiaires et à l'échelon le plus élevé qui est celui du dirigeant.

Une première conclusion qui s'impose au vu de ces résultats est donc celle de la non-fermeture complète du MIT. La seconde conclusion est qu'il paraît difficile de repérer au sein des coopératives des carrières conformes au modèle de carrière traditionnel.

Ceci nous amène à nous interroger sur les raisons de l'ouverture du MIT et nous rapproche du questionnement de Ballot et Piatecki (1996) sur la remise en cause de la cohérence du MIT lorsqu'il s'ouvre à l'extérieur. Pourquoi, alors même que les promotions internes des salariés de l'organisation auraient un effet incitatif (qui est l'une des fonctions du MIT), les coopératives recrutent-elles des candidats à l'extérieur? 
Les SCOP ont développé une logique de compétence, qui s'accorde plutôt bien avec la logique coopérative de promotion des hommes, et qui se mesure par l'impact relatif mais non négligeable de la formation dans l'accès au poste de dirigeant. Cependant, cette logique de compétence semble imposer un recours au marché externe chaque fois que la logique démocratique fondée sur le volontariat ne fait pas émerger au sein de la coopérative de successeur à un p.-d. g. en place depuis longtemps.

Comme Ballot et Piatecki (1996), nous pensons que les coopératives réalisent un arbitrage entre l'effet incitatif des promotions internes et la qualité requise des salariés dans les postes de l'échelon supérieur. En cas d'échec de la logique de compétence, la coopérative se trouve contrainte d'aller chercher des candidats extérieurs plus diplômés et donc plus productifs dans l'emploi supérieur que les salariés promus.

Mais là n'est pas, selon nous, le seul déterminant de cet arbitrage. Rappelons que l'idéal du projet coopératif fait clairement échec au principe de promotion hiérarchique comme méthode d'incitation sur deux points au moins. Premièrement, l'idéal coopératif s'oppose à la recherche de traits distinctifs liés au statut ou au pouvoir. La promotion à un poste plus élevé dans la hiérarchie n'est donc pas recherchée. Deuxièmement, l'existence de « tournois internes » mettant en concurrence des salariés potentiellement envisagés à la promotion n'est pas conforme à l'esprit égalitaire de la coopérative.

Ainsi, nous sommes amenées à réfuter en partie les conclusions auxquelles aboutissent Ballot et Piatecki grâce à leur modèle, qui affirment en effet que «lorsque le type d'organisation choisi est le marché interne, il est optimal de promouvoir à des emplois de cadre un certain nombre de travailleurs internes de préférence à des candidats externes mieux formés et ayant ainsi acquis des capacités d'organisateur supérieures » (p. 143). Mais, pour eux, le MIT ne peut avoir une fonction d'intégration d'une communauté d'individus autour d'un idéal sociétal ou professionnel.

Cette analyse contractualiste du MIT conduit à « une surévaluation des intérêts privés au détriment des solidarités dans l'entreprise »(Gomez, 1996, p. 152). Il est d'ailleurs intéressant de remarquer que lorsque Ballot et Piatecki envisagent de généraliser leur modèle à $n$ échelons, leur intuition leur indique que «le souci de la qualité doit l'emporter sur les préoccupations concernant les relations humaines quand il s'agit de pourvoir les postes de responsabilité » (p. 144).

Les motivations des coopérateurs se situant, selon l'esprit originel, ailleurs que dans la recherche de pouvoir et d'un rang hiérarchique plus élevé, il ne nous semble pas surprenant que le taux d'ouverture du MIT augmente quand on s'élève dans la hiérarchie d'une coopérative. 
L'enquête a révélé une fréquence élevée du renouvellement des mandats, évoquant l'hypothèse de l'existence d'une « carrière en tant que dirigeant » au sein de la SCOP. Dans un premier temps, nous avions interprété ce phénomène comme étant le résultat de la passivité des autres membres coopérateurs, quand la logique démocratique, fondée sur le volontariat, ne faisait pas émerger de successeur au sein de la coopérative. Nous pouvons nous interroger sur les raisons plus profondes de cette situation en mobilisant les analyses proposées par la théorie de l'enracinement en vue d'expliquer le comportement des dirigeants de SCOP. Ont-ils tendance à s'enraciner selon les stratégies précédemment décrites ?

\subsection{Des motifs d'enracinement diminués}

Quels moyens particuliers les dirigeants de coopératives mettent-ils en œuvre pour s'enraciner? Quels types d'investissements spécifiques privilégient-ils? Quels réseaux de capital humain développent-ils, pour conforter leur position par rapport aux partenaires dans la SCOP et pour diriger avec un certain degré de pouvoir discrétionnaire, qu'ils jugent nécessaire au nom de l'efficacité et de la réactivité pour la firme elle-même?

Notre réflexion nous pousse à émettre quelques réserves sur un certain nombre de points de la théorie. En premier lieu, il est important de noter que dans le cadre d'une coopérative, les actionnaires ou partenaires sont les salariés-coopérateurs qui travaillent dans l'entreprise (dans $80 \%$ des cas en moyenne). Par conséquent, ils peuvent exercer un contrôle indirect et informel sur le dirigeant, du fait même qu'ils participent aux activités productives. Ils peuvent mieux constater les effets de la stratégie comportementale du dirigeant et éventuellement la contester, en conseil d'administration, ou dans le quotidien. Il serait donc nécessaire de mieux cerner la nature et les modalités de contrôle du dirigeant dans une SCOP de manière à évaluer les risques de neutralisation des mécanismes disciplinaires par celui qui cherche à s'enraciner. En second lieu, la logique coopérative de solidarité et partage, associée à la notion «d'entrepreneur collectif », rend difficilement réalisable l'objectif de maximisation des rentes de la part du dirigeant. La transparence des grilles de rémunération, très resserrées d'ailleurs (de un à trois), pour l'ensemble des membres de la SCOP, y compris le dirigeant, ainsi que le mode de répartition des surplus entre tous, rendent inefficace toute stratégie visant à s'enrichir au détriment de ses partenaires.

En revanche, il est probable que le dirigeant de SCOP aura tendance, comme ailleurs, à accroître sa latitude managériale pour échapper à la «tyrannie » de la logique démocratique. Celle-ci l'obligerait à rendre compte quasiment en permanence de ses décisions devant le conseil d'administration ou l'assemblée générale des coopérateurs-salariés. Elle met en jeu à cette occasion le principe de révocabilité à tout instant, ou, a contrario, elle contraint le dirigeant à obtenir l'adhésion 
de tous (ou d'une majorité du moins) à ses projets, ainsi que l'approbation de ses décisions, au prix d'un temps important consacré à l'information et à l'explication auprès des coopérateurs.

Il est donc permis d'envisager d'une manière plus optimiste la renonciation, d'une part, à l'application stricte des principes de gestion démocratique avec révocabilité immédiate et, d'autre part, à l'objectif des membres coopérateurs de maximisation du revenu par personne. Le renouvellement des mandats devient un moyen d'accumuler du capital humain susceptible de produire de la valeur pour la firme en légitimant le pouvoir. Le rôle du conseil d'administration devient déterminant, car il aura à juger la performance du dirigeant et à déterminer le degré de latitude managériale qu'il est prêt à concéder pour préserver l'efficacité globale du fonctionnement de la coopérative.

Le prolongement du mandat au-delà de l'âge de la retraite est rarement observé et survient dans les situations limites où le successeur désigné n'est pas encore prêt à prendre seul les commandes. Ainsi, le système de la double commande se prolonge quelque temps. Il peut néanmoins arriver qu' un dirigeant refuse de prendre un «bras droit » malgré les pressions exercées par les membres coopérateurs pour qu'un successeur soit désigné afin de pallier les risques de disparition brutale. Ce type de comportement est plutôt en contradiction avec la logique coopérative.

Alors que la firme coopérative affiche toujours ses principes démocratiques comme moyen de lutter contre l'excès de pouvoir, nous pouvons nous interroger sur les « contre-pouvoirs » spécifiques au milieu coopératif qui permettent éventuellement de limiter les effets négatifs de l'enracinement du dirigeant de SCOP. Jusqu' où peut aller l'asymétrie d'information, qui risque de mener à l'illégitimité du pouvoir si le type d'investissements réalisés ne maximise pas la richesse collective ? Dans cette perspective, nous pensons que la formation au métier de dirigeant et à la vie coopérative, organisée dans le cadre de la confédération des SCOP, peut jouer ce rôle de générateur de concurrence interne, qui réduit le coût du changement de dirigeant, en créant des potentiels de remplacement ou de succession en interne. Elle permettrait ainsi de réduire le nombre de renouvellement de mandat pour un dirigeant et assurerait une meilleure circulation des mandats entre les salariés membres. Cependant, le p.-d. g. ne cèdera sa place que s'il est assuré de trouver au sein de la coopérative un poste adapté à ses compétences réelles, développées au cours de ses mandats, donc si l'organigramme offre de nouveaux créneaux d'emploi pour l'accueillir. Le cas contraire pourrait expliquer la stratégie d'enracinement.

\section{Pour un dépassement des théories contractualistes}

L'étude du mode d'accès au pouvoir dans les coopératives de production conduit à des conclusions partagées sur l'existence d'un véritable marché interne, puisque le modèle de l'autoproduction suivant un processus de promotion interne basé sur 
le volontariat cohabite avec le modèle de sous-traitance sur le marché externe. L'analyse du comportement du dirigeant, au cours de ses mandats successifs constituant une véritable carrière in situ, montre l'existence de barrières à la sortie avec des stratégies d'enracinement nuancées par la culture de ce type de firme.

Les théories en présence ne tiennent pas du tout compte du facteur éthique qui lie le dirigeant à la collectivité des membres de la coopérative. En effet, elles analysent le comportement du dirigeant au regard de sa seule motivation pour des stratégies favorables à son intérêt personnel. Seuls Castanias et Helfat (1992) envisagent une possible convergence d'intérêt entre le dirigeant et la firme. Et Charreaux (1996, p. 61) conclut qu' « un système économique dans lequel l'attitude des dirigeants est moins orientée vers l'opportunisme, en raison de normes sociales et culturelles, doit normalement se traduire par l'attribution d'un espace discrétionnaire plus étendu aux dirigeants et donc par une performance économique supérieure », ce qui est, à notre avis, le cas de la coopérative. La référence aux valeurs devient incontournable, d'autant que le comportement du dirigeant au cours de sa carrière ne s'explique que partiellement en termes d'enracinement pour augmenter son pouvoir discrétionnaire. La culture fait partie du rôle du dirigeant. Ensemble de normes, de valeurs, d'attitudes partagées, une culture forte est un moyen pour maintenir la cohésion interne.

Nous sommes proches de l'idée de «modèles mentaux » de Denzau et North (1993). Selon ce concept, les dirigeants prennent leurs décisions, non pas en fonction de la latitude discrétionnaire réelle dont ils disposent, mais de celle qu'ils perçoivent. Ses représentations sont influencées par un certain nombre de facteurs - personnalité des individus, contexte organisationnel, secteur d'activité, culture nationale -, et expliquent que des dirigeants peuvent avoir des réactions différentes face à des situations identiques. L'identité et l'origine de ces dirigeants, la définition de leur fonction et de l'entreprise vont structurer des «modèles mentaux » distincts.

Modèle mental et motivation au travail sont deux concepts liés si l'on définit la motivation comme «à la fois les raisons que les individus se donnent pour agir et la mobilisation de leur énergie individuelle pour atteindre ces buts » (Bernoux, 1995). Quels sont les objets motivants d'un dirigeant de coopérative? Quel sens ${ }^{3}$ donne-t-il à sa conduite, pour justifier qu'il se mobilise dans une action donnée ?

L'idée d'implication est inhérente au mode de fonctionnement démocratique de la coopérative puisqu'elle suppose d'inclure les individus dans les décisions qui les concernent et d'expliquer les raisons qui sous-tendent les décisions finales. L'un des grands principes de l'idéal coopératif réside dans la recherche d'une justice sur

3. Selon Bernoux (1995, p. 95), «le sens est dépendant des représentations des individus, de leur maturité affective et cognitive, de leurs expériences sociales et de leur situation au travail ». 
le lieu de travail, plus précisément d'une justice procédurale qui «s'attache aux mécanismes, procédures et processus à travers lesquels des décisions sont prises » (Rojot, 1997) ${ }^{4}$. L'implication, qui est une source de confiance et de coopération volontaire, engendre des comportements non utilitaristes et non opportunistes, et, par conséquent, modifie l'idée de pouvoir associée à la maîtrise du savoir.

Conceptuellement, 1'implication (commitment) se décline selon trois facteurs : une forte croyance et acceptation des buts et valeurs de l'organisation; la volonté de fournir des efforts considérables pour elle et une très forte intention de continuer à y appartenir (Porter, Mowday et Steers, 1979). La notion d'implication permet de «caractériser plus finement la relation entre la personne et l'institution » (Sire, 1992, p. 672), en prenant en considération à la fois les caractéristiques intrinsèques de l'individu (compétences, personnalité, système de représentation) et de l'organisation (les valeurs) et leurs projets mutuels de développement (Thévenet, 1992). La littérature consacrée à l'implication organisationnelle propose deux approches explicatives : l'approche instrumentale ou cognitive qui s'appuie sur les théories des avantages comparatifs et de l'échange, où l'individu calcule son adhésion à l'organisation et l'approche affective fondée sur l'attachement de la personne à l'entreprise, où elle s'engage à rester membre pour en atteindre les buts.

En référence à l'enquête réalisée en 1996, nous pouvons supposer que l'implication affective dominera l'implication cognitive chez les dirigeants de SCOP. En effet, parmi les valeurs les plus souvent citées comme les plus importantes, nous trouvons : l'épanouissement personnel, la possibilité de prendre des responsabilités, le respect d'un savoir-être et d'un savoir-faire et la convivialité. En moyenne $31 \%$ des dirigeants ont choisi de travailler dans une coopérative par «Idéal » (rang 1 dans $57 \%$ des cas), et $25 \%$ en raison de l' «Intérêt du poste » (rang 1 dans $38 \%$ des cas). Il semble donc que la firme coopérative permette l'accès à des niveaux de responsabilité managériale supérieurs aux firmes classiques en raison d'une forte culture qui valorise l'implication. D'autres études, décrites par Commeiras (1994), tendent à montrer par ailleurs que certaines variables démographiques sont corrélées avec l'implication, qui croît ainsi avec l'âge (plus de 50 ans), l'ancienneté et la position hiérarchique, résultats qui coïncident avec le profil des dirigeants de SCOP étudiées.

Par conséquent, l'implication constitue certainement a priori un des facteurs d'explication du mode d'enracinement du dirigeant.

Sur le plan théorique, le modèle contractualiste, dont Gomez (1996) souligne les limites, ne fournit donc aucune explication à deux aspects majeurs du fonctionnement des coopératives, à savoir les solidarités internes liées au sentiment

4. Justice procédurale et justice distributive s'opposent dans la mesure où la seconde s'attache uniquement aux résultats des décisions prises. 
d'appartenance et le phénomène d' « entrepreneur collectif » spécifique au statut de salarié associé. Le modèle conventionnaliste de l'entreprise pourrait à l'avenir nous inspirer plus directement pour la synthèse de nos enquêtes auprès des dirigeants de SCOP5.

\section{Bibliographie}

Alchian, A.A. et H. Demsetz (1972), « Production, information costs and economic organization », American Economic Review, vol. 62.

BALLOT, G. et C. PIATECKI (1996), «Le marché interne ouvert : un modèle », dans G. Ballot (dir.), Les marchés internes du travail : de la microéconomie à la macroéconomie, Paris, Presses universitaires de France, p. 121-146.

BAUER, M. et B. BERTIN-MOUROT (1992), Les 200. Comment devient-on un grand patron?, Paris, Le Seuil.

Berle, A. et G. Means (1932), The Modern Corporation and Private Property, New York, Harcourt, Brace and World, 380 p.

BernouX, P. (1995), La sociologie des entreprises, Paris, Le Seuil.

Biencourt, O., D. Eustache, Y. Gulllotin, T. Jolivet et P.Y. Steunou (1997), L'Adaptation de la gestion de l'emploi à l'évolution des marchés des produits, Rapport final pour le Commissariat du Plan, GAINS, Groupe d'analyse des itinéraires salariaux, Le Mans, Université du Maine, octobre, 109 p.

CADIN, L. (1997), «Faut-il sortir la GRH de ses frontières? », dans P. Besson, Dedans, dehors, Paris, Vuibert, p. 65-95.

CASTANias, R.P. et C.E. Helfat (1992), « Managerial and windfall rents in the market for corporate control », Journal of Economic Behavior and Organization, vol. 18.

Charreaux, G. (1996), «Pour une véritable théorie de la latitude managériale et du gouvernement des entreprises », Revue française de gestion, $\mathrm{n}^{\circ} 111$, novembredécembre, p. 50-64.

COMMEIRAS, N. (1994), «L'implication organisationnelle et contingences individuelles : les résultats d'une étude empirique », Actes du Congrès de l'Association francophone de la gestion des ressources humaines, Montpellier, p. 408-419.

5. L'enquête en cours en 1998-1999 sur un nombre restreint de cas de SCOP (une dizaine) nous permettra d'étudier en profondeur les différents aspects des carrières de dirigeants : les critères et les modalités de détection, sélection, formation, maintien et succession des «numéro un ». Le phénomène du renouvellement des mandats observé montre en effet une tendance à la confirmation de l'autorité et des compétences du dirigeant, alors que l'on pouvait craindre une restriction en raison du principe de la double qualité du coopérateur, à la fois intégré dans une ligne professionnelle et associé dans le processus décisionnel. 
DenZAu, A.T. et D.C. NORTH (1993), « Shared mental models : ideologies and institutions », Travail de recherche, Center for Politics and Economics, Claremont Graduate School et Center for the Study of Political Economy, Washington University (St. Louis), septembre.

DoERINGer, P.B. et M.J. PIORE (1971), International Labor Markets and Manpower Analysis, $2^{\mathrm{e}}$ édition, Sharpe, New York.

DUNLOP, J.T. (1966), «Job vacancy measures and economic analysis », dans The Measurement and Interpretation of Job Vacancy, A Conference Report of the National Bureau of Economic Research, National Bureau of Economic Research, New York, p. 27-47.

FAMA, E.F. (1980), «Agency problems and the theory of the firm », Journal of Political Economy, vol. 88.

Galambaud, B. (1993), "La gestion des cadres dirigeants et des dirigeants dans les entreprises françaises », Éducation permanente, $\mathrm{n}^{\circ} 114,1993-1$.

GARVEY, G.T. et P.L. SWAN (1994), «The economics of corporate governance : beyond the Marshallian firm », Journal of Corporate Finance, vol. 1, n ${ }^{\circ} 2$.

GLAIS, M. (1992), Économie industrielle, Paris, Litec.

GoMEZ, P.Y. (1996), Le gouvernement de l'entreprise, Paris, InterÉditions.

Huntzinger, F. (1994), «Forces et faiblesses du mouvement Scop dans la crise », Revue des études coopératives mutualistes et associatives (RECMA), $\mathrm{n}^{\circ} 253-254,3^{\mathrm{e}}$ et $4^{\mathrm{e}}$ trimestres, p. 38-47.

JENSEN, M.C. et W.H. MECKLing (1976), «Theory of the firm: managerial behavior, agency costs and ownership structure », Journal of Financial Economics, vol. 3.

KERR, C. (1954), « The balkanization of labor markets », dans E. Wight Bakke et al., Labor Mobility and Economic Opportunity, Cambridge, Massachusetts, Technology Press of MIT, p. 92-110.

MOYSAN-LOUAZEL, A. (1995), «Le modèle de carrière des ingénieurs à l'épreuve des mutations techniques et organisationnelles des entreprises », Revue Sciences de la Société, Toulouse, Presses universitaires du Mirail, no 36, octobre, p. 127-146.

MiLeS, R.E. et C.C. SNOW (1996), «Twenty-first-century careers », dans M.B. Arthur et D.M. Rousseau, The Boundaryless Career : A New Employment Principle for a Nex Organizational Era, New York, Oxford University Press, p. 97-116

PAQUeRot, M. (1996), «L'enracinement des dirigeants et ses effets », Revue française de gestion, $\mathrm{n}^{\mathrm{0}} 111$, novembre-décembre, p. 212-225.

Porter, L.W., R.T. MoWday et R.M. SteERs (1979), «The measurement of organizational commitment », Journal of Vocational Behavior, vol. 14, p. 224-247.

Rojot, J. (1997), «Qu'est-ce que la justice au travail ?», L'Expansion et Management Review, décembre, p. 25

SHLEIFER, A. et R.W. VISHNY (1989), «Manager entrenchment: the case of manager specific investment », Journal of Financial Economics, vol. 2.

Simon, H. (1980), Le nouveau management, Paris, Economica. 
SIRE, B. (1992), « De la flexibilité potentielle à la flexibilité effective par la prise en compte des motivations », dans A. Labourdette (dir.), Mélanges en l'honneur de Jean-Guy Mérigot, Paris, Economica, p. 665-677.

STIGLITZ, J.E. et A.S. EDLIN (1992), «Discouraging rivals : managerial rent seeking and economic insufficiencies », NBER Working-Paper Series, $\mathrm{n}^{\circ} 4145$.

THÉVEnET, M. (1992), Impliquer les hommes dans les entreprises, Paris, Éditions Liaisons sociales.

Williamson, O.E (1964), The Economics of Discretionary Behavior: Managerial Objectives in a Theory of Firm, Englewood Cliffs, N.J., Prentice-Hall, 182 p.

Williamson, O.E. (1985), The Economics Institutions of Capitalism, New York, Free Press, $450 \mathrm{p}$. 